\title{
Calcium/Calmodulin-Dependent Protein Kinase Type II Subunit Beta
}

National Cancer Institute

\section{Source}

National Cancer Institute. Calcium/Calmodulin-Dependent Protein Kinase Type // Subunit

Beta. NCl Thesaurus. Code C104832.

Calcium/calmodulin-dependent protein kinase type II subunit beta (666 aa, $\sim 73 \mathrm{kDa}$ ) is encoded by the human CAMK2B gene. This protein plays a role in calcium signaling in the central nervous system. 